\title{
Body sway and vibration perception thresholds in normal aging and in patients with polyneuropathy
}

\author{
P S Bergin, A M Bronstein, N M F Murray, S Sancovic, K Zeppenfeld
}

\begin{abstract}
Body sway and vibration perception in the lower limbs were measured in 32 normal subjects and 25 patients with peripheral neuropathies; nerve conduction studies were also performed in the patients with neuropathies. Body sway was measured by means of force-plate posturography, and three methods were used to assess vibration perception: a neurothesiometer, a semiquantitative tuning fork, and the bone vibrator of a conventional audiometer. Body sway and vibration perception were increased in the patients with peripheral neuropathies, and there was significant correlation between these measures. These findings, together with the lack of correlation between sway and muscle strength, indicate that the main source of unsteadiness in these patients is the loss of proprioceptive information. Vibration perception and body sway did not correlate with the electrophysiological variables, indicating that these measures assess different aspects of peripheral nerve function. In all subjects there was close correlation between vibration perception as assessed by the neurothesiometer and the audiometer, indicating that the audiometer could be used to screen proprioceptive function in patients with balance disorders. In normal subjects age correlated with vibration perception (measured with the neurothesiometer and audiometer) and also with body sway standing on foam. This suggests that the increased body sway in elderly people may partly be due to reduced proprioception in the lower limbs.
\end{abstract}

$(\mathcal{F}$ Neurol Neurosurg Psychiatry 1995;58:335-340)

Keywords: polyneuropathy; body sway; vibration perception; aging

Postural control relies on information provided by the visual, vestibular, and proprioceptive systems. Severe disturbances of balance and gait can occur in proprioceptive afferent disorders (for example, tabes dorsalis, ${ }^{12}$ polyneuropathies ${ }^{3-6}$ ). There have, however, been few attempts to quantify the degree of unsteadiness in patients with neuropathies, or to correlate this with the severity of the neuropathy.
Proprioceptive signals and vibration sensation are conveyed by large fibres of peripheral nerves, ${ }^{7}$ and these are also the fibres that are stimulated during routine nerve conduction studies. ${ }^{8}$ It is not known whether the degree of unsteadiness is related to vibration thresholds in patients with neuropathies, nor how it relates to the electrophysiological functional status of the peripheral nerves. Changes due to normal aging also cause deterioration in both postural control and vibration perception, ${ }^{9-13}$ but it is not clear whether these changes are related, and if so, under what conditions.

Neuropathies may give rise to subtle off balance sensations, and presumably they may also delay the process of compensation of vestibular lesions. ${ }^{14}$ Patients with proprioceptive disorders may therefore present to a neuro-otology clinic complaining of poor balance. Because an audiometer includes a bone vibrator, it should be possible to use this to assess proprioceptive function. By using an audiometer in this way to screen for neuropathies, it may be possible to perform a more thorough assessment of balance function in patients attending audiology or otology clinics.

In this study we correlated data from forceplate posturography with three methods of assessing vibration perception: a semiquantitative tuning fork, a neurothesiometer, and the bone vibrator of a conventional audiometer. We also assessed how age affects vibration perception and postural control. Finally, we determined whether vibration perception and postural control are related to nerve conduction variables in patients with peripheral neuropathies.

\section{Methods}

Thirty two normal subjects (mean age 42 (SD 18) years; range $24-85$ years) and 25 patients who had peripheral neuropathies (mean age 55 (SD 14) years; range 27-86 years) were studied. The clinical severity of the patients studied was limited by the fact that they had to be able to stand unaided on the sway platform. Patients with additional neurological or labyrinthine symptoms or signs were excluded. All subjects had vibration thresholds determined by the three different methods, and all had body sway assessed by force-plate posturography. Vibration thresholds were measured because they are easily quantified and also because they include a temporal component likely to be relevant to 
the dynamic control of postural sway. All patients with neuropathies had nerve conduction tests performed, although controls did not. Standard neurophysiological criteria were used to determine whether a neuropathy was present, and whether it was axonal or demyelinating. ${ }^{15}$

Vibration thresholds were measured with a neurothesiometer (Horwell Ltd, London), which vibrates at $100 \mathrm{~Hz}$, a conventional audiometer (Peters AP.5 mk II, Sheffield) vibrating at $250 \mathrm{~Hz}$, and a $64 \mathrm{~Hz}$ tuning fork (Aesculap, Germany). Vibration sense was determined at both ankles (medial malleoli) and at both tibial tuberosities. The vibrators were applied maintaining as constant a pressure as possible at the stimulating site; with the neurothesiometer this was easily achieved by using the weight of the instrument. More variability may have occurred with the audiometer or tuning fork as with these instruments the pressure had to be manually generated by the experimenter. With both the neurothesiometer and audiometer, the procedure to determine thresholds was as follows. To demonstrate the sensation of vibration, the subject was given a suprathreshold stimulus in a symptom free region. The vibrator was then applied to the test site and the amplitude was gradually increased from zero until the subject signalled that vibration was just perceived; five measurements were taken from each site and the median value was used for this study. This tuning fork was chosen because it allowed a semiquantitative assessment; the two vibrating prongs bore eight numbered marks that become visible at specific amplitudes of vibration. The tuning fork was vibrated maximally and then applied until the subject signalled that vibration was no longer perceived, at which point the corresponding number on the fork was noted by the examiner.

Body sway was measured as the displacement of the centre of foot pressure in the anterior-posterior direction detected by a rigid platform mounted on strain gauges. Body weight was normalised to $70 \mathrm{~kg}$. The total length (mm) travelled by the centre of foot pressure in 60 seconds was calculated as described in previous publications. ${ }^{216}$ Subjects were tested in four conditions, lasting one minute each: standing directly on the platform with eyes open and closed, and standing on foam rubber with eyes open and closed. The Romberg coefficient was calculated as sway with eyes open/sway with eyes closed. When subjects were tested with the eyes open, they were instructed to maintain optic fixation straight ahead on a large map fixed to one of the walls of the normally lit experimental room; eye fixation point distance was $50 \mathrm{~cm}$.

Nerve conduction studies were performed on either a Medelec MS6 or a Nicolet Viking 11 electromyograph. The nerves studied varied according to the specific problem for which the patient had been referred: all patients had both sensory and motor studies, including sensory studies of sural and median nerves, and motor studies of one lateral
Table 1 Neuropathy score

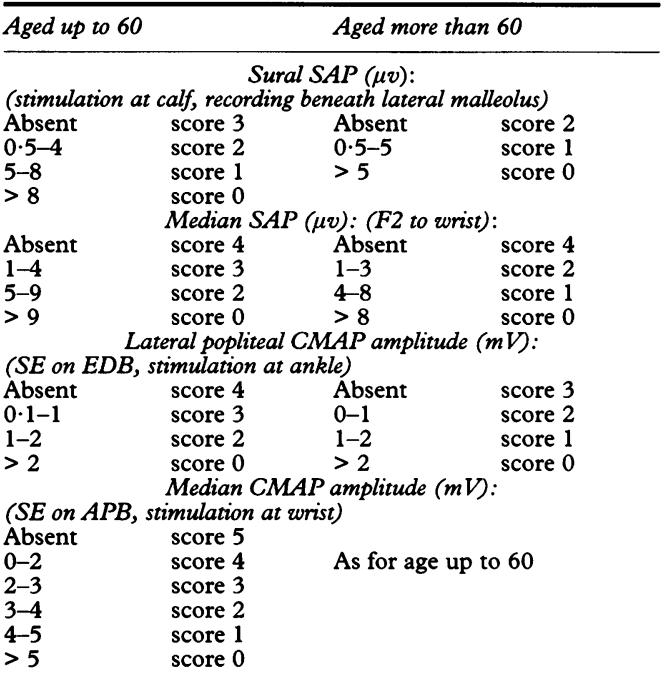

The amplitude of the SAP or CMAP determined the score for The amplitude of the SAP or CMAP determined the score for compute a composite neuropathy score. (When both left and right nerves were studied, then a mean score for that nerve was calculated.) Two points were added to the total if an upper limb motor conduction velocity was less than $40 \mathrm{~m} / \mathrm{s}$, or a lower limb motor conduction velocity was less than $35 \mathrm{~m} / \mathrm{s}$. $\mathrm{SAP}=$ sensory action potential; CMAP = compound muscle action potential; $\mathrm{F} 2=$ finger $2 ; \mathrm{SE}=$ surface electrode $; \mathrm{EDB}=$ extensor digitorum brevis; APB = abductor pollicis brevis.

popliteal nerve. All but one had an upper limb motor study performed; in this particular patient the median sensory action potential was normal. Standard electrode positions for recording and stimulation were used. Sixteen patients had axonal neuropathies, eight had demyelinating neuropathies and one had a mixed picture. Most patients also had EMGs of the tibialis anterior. Lower limb strength was assessed according to the MRC scale.

To grade the electrophysiological severity of the neuropathy, we devised a composite "neuropathy score" (table 1). The number of points allocated for each nerve depended on the amplitude of the sensory action potential or compound muscle action potential, and indicated the severity of the underlying neuropathy. Two points were added to the total if an upper limb motor conduction velocity was less than $40 \mathrm{~m} / \mathrm{s}$, or a lower limb motor conduction velocity was less than $35 \mathrm{~m} / \mathrm{s}$. Scoring differed slightly for patients older than 60 .

As patients with slowed conduction may have desynchronisation of nerve volleys with consequent reduction in vibration thresholds, we also determined if there was any correlation between vibration thresholds and median nerve motor conduction velocities.

Statistical analysis was based on the nonparametric Mann-Whitney test and the Spearman rank correlation coefficient (Srcc) from the package CSS Statistica. Significance level was set at a value of $P \leqslant 0.01$.

\section{Results}

VIBRATION THRESHOLDS

Tables 2 and 3 show the data for the patients and the normal subjects for all variables measured. Patients had significantly higher 
Table 2 Vibration perception and body sway in normal controls and patients with polyneuropathy

\begin{tabular}{|c|c|c|c|c|}
\hline & $\begin{array}{l}\text { Normal controls } \\
(n=32) \\
\text { Mean }(S D)\end{array}$ & $\begin{array}{l}\text { Patients } \\
(n=25) \\
\text { Mean }(S D)\end{array}$ & $\begin{array}{l}\text { Significance } \\
\text { Z Value }\end{array}$ & $p$ Value \\
\hline \multicolumn{5}{|l|}{ Neurothesiometer $(V)$ : } \\
\hline Right ankle & $12 \cdot 5(9 \cdot 2)$ & $30 \cdot 8(16 \cdot 0)$ & $4 \cdot 2$ & $<0.001$ \\
\hline Left ankle & $12 \cdot 8(9 \cdot 0)$ & $28 \cdot 5(16 \cdot 1)$ & $3 \cdot 7$ & $<0.001$ \\
\hline Right tibia & $14 \cdot 4(8 \cdot 2)$ & $27 \cdot 8(15 \cdot 7)$ & $3 \cdot 5$ & $<0.001$ \\
\hline Left tibia & $13 \cdot 8(8 \cdot 4)$ & $26 \cdot 5(14 \cdot 4)$ & $3 \cdot 5$ & $<0.001$ \\
\hline \multicolumn{5}{|l|}{ Audiometer (dB): } \\
\hline Right ankle & $36 \cdot 4(12 \cdot 2)$ & $55 \cdot 0(14 \cdot 2)$ & $4 \cdot 7$ & $<0.001$ \\
\hline Left ankle & $35 \cdot 6(13 \cdot 8)$ & $55 \cdot 6(14 \cdot 3)$ & $4 \cdot 6$ & $<0.001$ \\
\hline Right tibia & $34.9(13.7)$ & $52 \cdot 3(15 \cdot 2)$ & $4 \cdot 1$ & $<0.001$ \\
\hline Left tibia & $35 \cdot 2(13 \cdot 2)$ & $53 \cdot 4(14 \cdot 8)$ & $4 \cdot 4$ & $<0.001$ \\
\hline \multicolumn{5}{|l|}{ Tuning fork (arbitrary units): } \\
\hline Right ankle & $7 \cdot 9(0 \cdot 7)$ & $4 \cdot 6(3 \cdot 0)$ & $3 \cdot 2$ & 0.001 \\
\hline Left ankle & $7 \cdot 9(0.4)$ & $4 \cdot 7(3.0)$ & $3 \cdot 2$ & 0.001 \\
\hline Right tibia & $7 \cdot 8(0 \cdot 8)$ & $4.9(2.5)$ & $3 \cdot 0$ & 0.002 \\
\hline Left tibia & $7.9(0.5)$ & $5 \cdot 2(2 \cdot 8)$ & $4 \cdot 4$ & 0.001 \\
\hline \multicolumn{5}{|l|}{ Body sway $(\mathrm{mm} / \mathrm{min})$ : } \\
\hline Eyes open & $145 \cdot 2(43 \cdot 4)$ & $497 \cdot 2(678 \cdot 7)$ & $4 \cdot 9$ & $<0.001$ \\
\hline Eyes closed & $280 \cdot 4(156 \cdot 5)$ & $1150 \cdot 3(1179 \cdot 5)$ & $5 \cdot 1$ & $<0.001$ \\
\hline $\begin{array}{l}\text { Romberg } \\
\text { (eyes closed/eyes open) }\end{array}$ & $1.9(0.6)$ & $2 \cdot 7(1 \cdot 0)$ & $3 \cdot 6$ & $<0.001$ \\
\hline Foam: eyes open & $178 \cdot 1(66 \cdot 7)$ & $468 \cdot 7(576 \cdot 5)$ & $4 \cdot 1$ & $<0.001$ \\
\hline Foam: eyes closed & $356.8(128.9)$ & $1118 \cdot 7(790 \cdot 7)$ & $5 \cdot 3$ & $<0.001$ \\
\hline Foam: Romberg & $2 \cdot 1(0 \cdot 6)$ & $3.0(1 \cdot 2)$ & $2 \cdot 9$ & 0.003 \\
\hline
\end{tabular}

thresholds for vibration perception than normal controls.

For all subjects, vibration thresholds correlated across both sides of the body (for example, right and left ankle, Srcc $=0.846, \mathrm{P}<$ 0.001 ) and for different sites (for example, right ankle and right tibia, Srcc $=0.813, \mathrm{P}<$ $0 \cdot 001$ ). Thus unless specified data used are from the right ankle only.

In normal subjects, vibration threshold determined by the neurothesiometer and the audiometer were highly correlated $(\mathrm{Srcc}=$ $0.864, P<0.001$; fig 1 ); however, neither of these measures correlated with measurements obtained with the tuning fork ( $\operatorname{Srcc}=0 \cdot 147$, NS).

In patients there was also significant correlation between measurements with the audiometer and the neurothesiometer $(\mathrm{Srcc}=$ $0.858, \mathrm{P}<0.001$; fig 1$)$. By contrast with the normal subjects, there was also correlation between these measurements and thresholds determined with the tuning fork $(\mathrm{Srcc}=$ $-0.603, P=0.001$ ).

There was no significant difference in

Table 3 Vibration perception, body sway, and nerve conduction studies in patients with axonal or demyelinating neuropathy

\begin{tabular}{|c|c|c|c|c|}
\hline & $\begin{array}{l}\text { Axonal } \\
(n=16) \\
\text { Mean (SD) }\end{array}$ & $\begin{array}{l}\text { Demyelinating } \\
(n=8) \\
\text { Mean (SD) }\end{array}$ & $\begin{array}{l}\text { Significance } \\
\text { Z Value }\end{array}$ & p Value \\
\hline Neurothesiometer (V) & $31 \cdot 8(16 \cdot 5)$ & $25.9(14.5)$ & 0.9 & 0.4 \\
\hline Audiometer (dB) & $57 \cdot 3(13 \cdot 6)$ & $48 \cdot 6(14 \cdot 0)$ & $1 \cdot 3$ & $0 \cdot 2$ \\
\hline Tuning fork (arbitrary units) & $3.9(3 \cdot 0)$ & $6.4(1.9)$ & $2 \cdot 0$ & $0 \cdot 1$ \\
\hline \multicolumn{5}{|l|}{ Body sway $(\mathrm{mm} / \mathrm{min})$ : } \\
\hline Eyes open & $269 \cdot 0(118 \cdot 0)$ & $970.0(1082 \cdot 8)$ & $1 \cdot 1$ & $0 \cdot 3$ \\
\hline Eyes closed & $782 \cdot 0(443 \cdot 0)$ & $1864.0(1858.4)$ & $1 \cdot 1$ & $0 \cdot 3$ \\
\hline $\begin{array}{l}\text { Romberg } \\
\text { (eyes closed/eyes open) }\end{array}$ & $2 \cdot 8(1 \cdot 2)$ & $2 \cdot 4(0 \cdot 7)$ & $0 \cdot 2$ & 0.9 \\
\hline Foam: eyes open & $313.0(163.0)$ & $851 \cdot 6(982 \cdot 3)$ & $1 \cdot 2$ & $0 \cdot 2$ \\
\hline Foam: eyes closed & $946 \cdot 0(440 \cdot 0)$ & $1453 \cdot 3(1294 \cdot 0)$ & 0.2 & 0.9 \\
\hline Foam: Romberg & $3 \cdot 2(1 \cdot 1)$ & $2 \cdot 2(0.9)$ & $2 \cdot 1$ & 0.03 \\
\hline Score & $6.9(2 \cdot 8)$ & $11 \cdot 3(4 \cdot 1)$ & $2 \cdot 1$ & 0.03 \\
\hline \multicolumn{5}{|l|}{ Median nerve: } \\
\hline $\begin{array}{l}\text { Conduction velocity (m/s) } \\
\text { Distal latency (ms) }\end{array}$ & $\begin{array}{l}48 \cdot 6(10 \cdot 5) \\
4 \cdot 8(2 \cdot 7)\end{array}$ & $\begin{array}{c}24 \cdot 2(10 \cdot 0) \\
8 \cdot 1(4 \cdot 2)\end{array}$ & $\begin{array}{l}3 \cdot 4 \\
2 \cdot 8\end{array}$ & $\begin{array}{r}<0.001 \\
0.004\end{array}$ \\
\hline Muscle power (MRC scale) & $4 \cdot 3(0 \cdot 8)$ & $4 \cdot 3(0 \cdot 4)$ & $0 \cdot 7$ & 0.5 \\
\hline
\end{tabular}

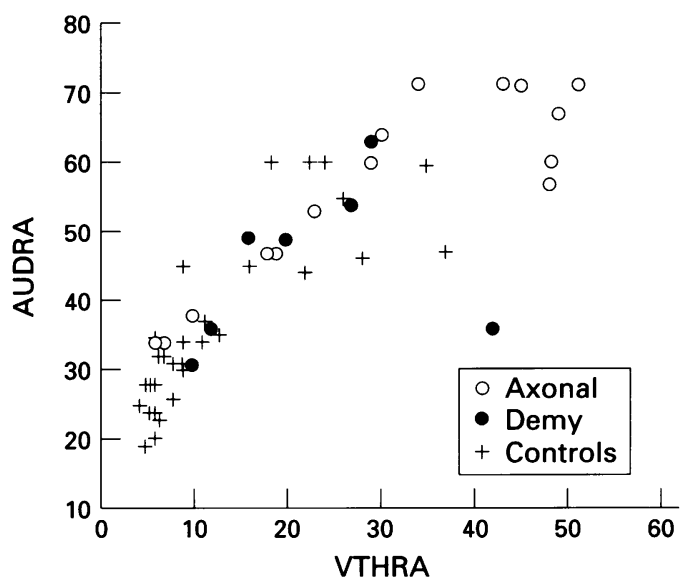

Figure 1 Scatterplot of vibration thresholds measured at the right ankle with the neurothesiometer in "volt units" (VTHRA) and with the audiometer in $\mathrm{DB}$ (AUDRA) for patients with axonal and demyelinating neuropathies, and for normal controls. vibration perception between patients with axonal or demyelinating neuropathies (table 3 , fig 1).

\section{BODY SWAY AND RELATION TO VIBRATION THRESHOLDS}

In the normal subjects there was only partial correlation between body sway and vibration perception: there was some correlation between vibration thresholds and body sway while standing on the platform with eyes closed $(\mathrm{Srcc}=0.427, \mathrm{P}=0.014)$. There was better correlation between vibration thresholds and body sway while standing on the foam, both with eyes open (Srcc $=0 \cdot 679, \mathrm{P}<$ $0.001)$ and closed (Srcc $=0.611, P<0.001)$. There was no significant correlation between vibration thresholds and sway with eyes open while standing directly on the platform (Srcc $=0.222$, NS). Nor was there correlation between thresholds and Romberg quotients on the platform ( $\mathrm{Srcc}=0.278$, NS) or on foam (Srcc $=-0 \cdot 183, \mathrm{NS}$ ).

As expected, body sway with eyes open and closed were correlated $(\mathrm{Srcc}=0.717, \mathrm{P}<$ $0.001)$. Sway with eyes open was not correlated with the Romberg quotient (Srcc= 0.041 , NS) but body sway with eyes closed was correlated (Srcc $=0.661, P<0.001)$. This indicates that large Romberg quotients are mainly due to enhanced body sway with eyes closed.

In all conditions, patients swayed significantly more than controls (table 2, figs 2A, B). There was no significant difference in body sway between patients with axonal or demyelinating neuropathies (table 3 , figs $2 \mathrm{~A}$, B). Body sway did not correlate with muscle power.

By contrast with the findings in normal subjects, vibration thresholds in patients were well correlated with body sway in all conditions - that is, standing on the platform with eyes open $(\mathrm{Srcc}=0.503, \mathrm{P}=0.01)$, and closed (Srcc $=0.563, P=0.003)$ and standing on 
Figure 2 Scatterplots showing vibration thresholds at the right ankle with the neurothesiometer in volt units (VTHRA) and body sway in $\mathrm{mm} / \mathrm{min}$ with eyes open (BSEO; figure $2 A$ ) and with eyes closed (BSEC; figure 2B). All subjects are included. (note the logarithmic scale for body sway ( $y$ axis, figures $2 A$ and $2 B$ ).
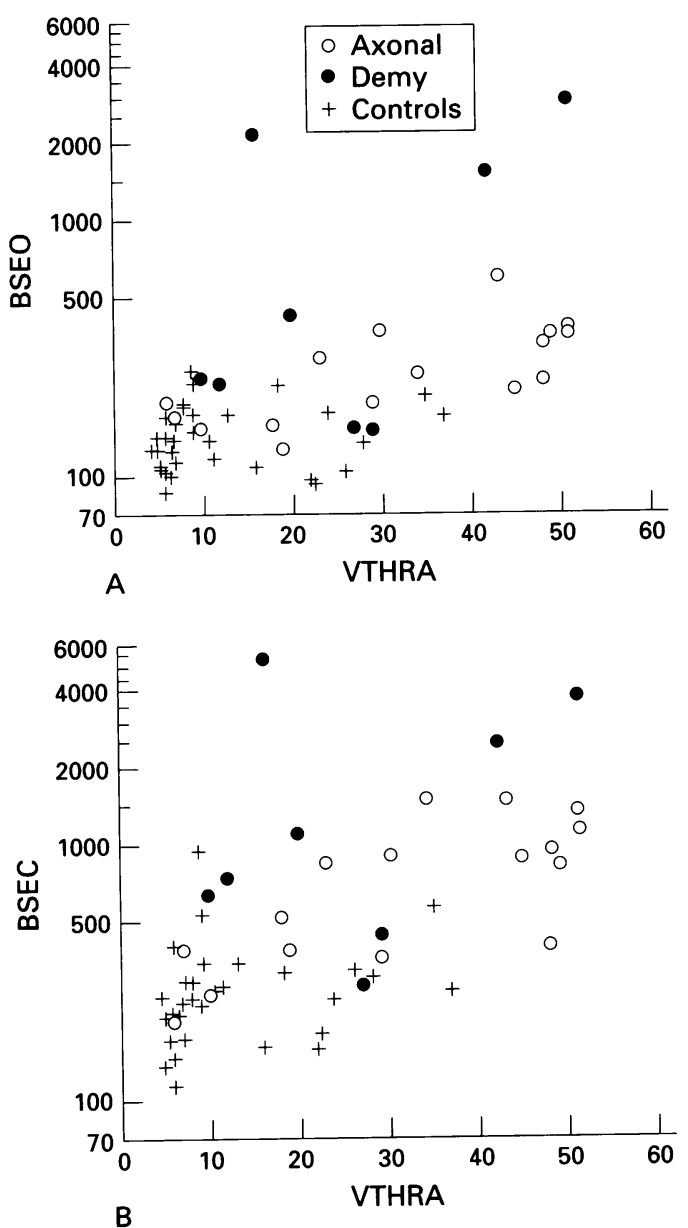

foam (eyes open Srcc $=0.524, P=0.008$; eyes closed Srcc $=0.531, P=0.07$ ). This correlation seemed to depend on the findings in the axonal group of neuropathies as it was higher in these (Srcc $=0.690$ to $0.730, \mathrm{P}<$ 0.003 in all four conditions; figs $2 \mathrm{~A}, \mathrm{~B}$ ) and non-significant in the demyelinating group (Srcc $=0.007$ to 0.261 ; figs $2 \mathrm{~A}, \mathrm{~B}$ ). In patients, there were no significant correlations between vibration thresholds and the Romberg quotient.

AGE EFFECT

Vibration thresholds measured with the neurothesiometer and with the audiometer

Figure 3 Three dimensional scatterplot correlating years of age, vibration thresholds (in volt units) with the neurothesiometer at the right ankle (VTHRA), and body sway (in $\mathrm{mm} / \mathrm{min}$ ) on the foam with eyes open (BSFEO) in normal subjects. increased with age in the normal subjects (neurothesiometer: Srcc $=0.705, P<0.001$; audiometer: $\operatorname{Srcc}=0.744, \mathrm{P}<0.001$; fig 3; $\mathrm{x}-$ $\mathrm{y}$ axes). Tuning fork measurements were not correlated with age $(\mathrm{Srcc}=-0 \cdot 209, \mathrm{NS})$.

Age and body sway were positively correlated when normal subjects stood on foam (eyes open: $\operatorname{Srcc}=0.636, P=0.001$; eyes closed: $\operatorname{Srcc}=0.522, P=0.002 ;$ fig $3 ; x-z$ axes) but not while they stood directly on the platform (eyes open: $\operatorname{Srcc}=0 \cdot 123, \mathrm{NS}$; eyes closed: Srcc $=0 \cdot 226$, NS). A multiple regression analysis was carried out with age as the dependent variable, testing correlation with vibration thresholds (neurothesiometer and audiometer), and body sway (standing on platform and foam, eyes open and closed). This multiple relation was highly significant $(r$ $=0.864 F=11.80, P<0.001)$; the major determinant of this correlation with age was vibration threshold as measured with the neurothesiometer $(\beta=0.738, \mathrm{P}<0.001)$.

In patients, none of the measures of vibration sensation or body sway correlated with age.

ELECTROPHYSIOLOGY AND RELATION TO VIBRATION THRESHOLDS AND BODY SWAY Neuropathy scores ranged from 2 to 15 (mean $7 \cdot 8)$. There was a striking lack of correlation ( $P>0.05)$ between the electrophysiological variables (neuropathy score and conduction velocity) and each of the functional measurements (vibration perception with any technique or site, sway in any condition, and muscle strength). This lack of correlation is not due to some inconsistency in either the EMG or the functional studies, because the EMG variables are interrelated (neuropathy score-conduction velocity, $\operatorname{Srcc}=-0.668, P$ $<0.001)$. As mentioned, the functional studies are similarly correlated between themselves.

\section{Discussion}

Force-plate measurements confirmed that patients with neuropathies have increased postural sway. Although expected, this finding has rarely been reported in quantitative form. ${ }^{17-19}$ Sway correlated with vibration perception but not with muscle strength. Assessment of muscle power was only a semiquantitative clinical estimate, but the results strongly suggest that it is lack of proprioception, rather than weakness, that causes patients to be unsteady.

The finding that patients differed from controls in all sway testing conditions indicates that visual input cannot fully compensate for the impairment in proprioception, even when subjects stand on a firm surface. This is unlike patients with total loss of vestibular function ${ }^{20}$ and is further evidence that proprioception is the single most important sensory input for postural control in humans. Unfortunately, this fact is not always appreciated in the assessment of patients with balance disorders.

In the control group vibration perception 
was only correlated with body sway when standing on foam or with eyes closed. This contrasts with the patients, in whom vibration thresholds correlated with sway under all conditions. These findings suggest that when the proprioceptive system is stressed (for example, darkness or compliant supporting surface) small differences in vibration perception in the normal population may become important for sway control. In such conditions, subjects with lower thresholds sway less than those with higher thresholds.

This finding is particularly relevant to the problem of unsteadiness in the normal elderly population. In our sample, age, raised vibration thresholds, and increased postural sway were interrelated, although multiple regression analysis indicated that the most significant association was between age and vibration thresholds. Furthermore, the data showed that sway increased with age when the subjects stood on foam but not on the firm platform. This shows that the effect of age on postural sway becomes manifest when taxing conditions are met. Vibration perception and body sway are also correlated only when the postural system is somewhat stressed. Taken together, these findings emphasise the role of decreased proprioception in the balance disorder of the elderly.

One of our aims was to compare vibration perception with peripheral nerve conduction variables in patients with neuropathies. To do this, we had to devise a means of grading the severity of the neuropathy in electrophysiological terms. Clinical severity of polyneuropathies would not be satisfactory as many of the most disabling features (for example, autonomic involvement, disturbed bulbar function, trophic changes, pain) are unrelated to somatic afferent function in large fibres. We devised our neuropathy score because we are not aware of any simple neurophysiological system for grading neuropathies, although a more complex method has since been devised. ${ }^{21}$ It is clearly unsatisfactory to equate the severity of a neuropathy with the result of any single test. For example, although the amplitude of the sural action potential is particularly valuable in confirming the presence of a neuropathy, not all patients with an absent sural action potential have a neuropathy of similar severity. Broadly, a patient with absent sural and median action potentials has a more severe neuropathy than one with an absent sural and a median sensory action potential of $10 \mu \mathrm{v}$, whereas a patient with abnormal motor conduction and absent sensory potentials is more affected than one with sensory involvement alone. In addition, normal ranges tend to decline with age. For these reasons we produced summary scores, based on amplitudes of both sensory and motor nerves in upper and lower limbs, which took age into account. There is also an additional difficulty, which arises from grading both demyelinating and axonal neuropathies on the same scale. For example, one patient included in this study had severe slowing of motor nerve conduction, but normal sensory and compound muscle action potentials. We therefore added two points to the total score if the conduction velocity fell below set values.

We did not, in fact, find any correlation between the neuropathy score and the more functional measures of body sway, vibration thresholds, or muscle power. Nor was there any correlation between sway or vibration and conduction velocities. Perhaps this is not totally surprising, as clinical experience suggests that the degree of abnormality apparent on EMG does not necessarily correlate with symptoms, ${ }^{3}$ and relatives of patients with hereditary neuropathies may be totally asymptomatic while having unequivocal abnormalities of nerve conduction. ${ }^{22}$ Some of our patients with demyelinating neuropathiesfor instance, hereditary sensorimotor neuropathy type 1 , had absent sensory potentials and profoundly slowed conduction, yet their vibration thresholds were only slightly increased. This contradicts conventional wisdom, which states that impaired sensation of vibration is usually found in demyelinating neuropathies, even when patients are asymptomatic. ${ }^{23}$ Our findings suggest that nerve conduction studies are not a good indicator of functional status, despite being a very useful diagnostic tool. Presumably, transducing vibratory stimuli or signalling limb or joint position or motion requires temporospatial summation over large areas for a considerable time. This is in sharp contrast to the highly synchronised volley elicited by the supramaximal electrical stimuli used during nerve conduction studies. Thus tests of body sway and vibration perception on the one hand, and tests of nerve conduction on the other, seem to measure different aspects of peripheral nerve function.

A practical point to note is that vibration thresholds as measured by the audiometer correlated well with the measurements from the neurothesiometer. This indicates that an audiometer could also be used to screen patients for a neuropathy. Such screening could be of particular value in balance disorder clinics biased towards otological assessment where measurement of proprioceptive function may not be routinely undertaken. At present, however, there is little evidence to indicate whether changes in lower limb proprioceptive function contribute to subtle sensations of unsteadiness or retard the process of compensation of vestibular disorders. ${ }^{14}$

The lack of correlation with the tuning fork measurements in healthy controls suggests that the resolution of the tuning fork is not enough to detect the small variations in perception of vibration present within the normal population. The tuning fork is sufficiently sensitive, however, to detect the relatively gross differences in the neuropathic patients, as there was correlation between all three techniques of measuring vibration perception in this group. The introduction of semiquantitative scales, such as the one provided by the tuning fork used in this study, improves the quality of the bedside clinical assessment of vibration perception. 
We express our gratitude to Dr P Haughton (Hull Royal Infirmary) for kindly providing the force platform, and Dr CJ Fowler (National Hospital for Neurology and Neurosurgery) for early advice on vibration perception techniques.

1 Adams R, Victor M. Principles of neurology. 5th ed. New York: McGraw Hill, 1993:599-639.

2 Bronstein AM. Supression of visually evoked postural responses. Exp Brain Res 1986;63:655-8.

3 Thomas PK. Clinical features and differential diagnosis. In: Dyck PJ, Thomas PK, Lambert EH, Bunge R, In: Dyck PJ, Thomas PK, Lambert EH, Bunge R,
eds. Peripheral neuropathy. 2nd ed. Philadelphia: WB eds. Peripheral neuropathy.

4 Denny-Brown D. Primary sensory neuropathy with muscular changes associated with carcinoma. If Neurol Neurosurg Psychiatry 1948;11:73-87.

5 Sterman AB, Schaumburg HH, Asbury AK. The acute sensory neuronopathy syndrome; a distinct clinical entity. Ann Neurol 1980;7:354-8.

6 Griffin JW, Cornblath DR, Alexander E, et al. Ataxic sensory neuropathy and dorsal root ganglionitis associated with Sjogrens syndrome. Ann Neurol 1990;27:304-15.

7 Light AR, Perl ER. Peripheral sensory systems. In: Dyck PJ, Thomas PK, Lambert EH, Bunge R, eds. Peripheral neuropathy. 2nd ed. Philadelphia: WB Saunders, 1984: 210-30.

8 Dorfman LJ. AAEM Minimonograph 21: the distribution of conduction velocities in peripheral nerves: a review. of conduction velocities in

9 Kollegger H, Baumgartner C, Wober C, Oder W, Deecke L. Spontaneous body sway as a function of sex, age, and vision: posturographic study in 30 healthy adults. Eur Neurol 1992;32:253-9.

10 Steinberg FU, Graber AL. The effect of age and peripheral circulation on the perception of vibration. Arch Phys Med Rehabil 1963;44:645-50.

11 Bloom S, Till S, Sonsken P, Smith S. Use of a biothesiometer to measure individual vibration thresholds and their variation in 519 non-diabetic subjects. $B M \mathcal{F} 1984$; 288: 1793-5.
12 Merchut MP, Toleikis SC. Aging and quantitative sensory thresholds. Electromyogr Clin Neurophysiol 1990;30: 293-7.

13 Wiles PG, Pearce SM, Rice PJS, Mitchell JMO. Vibration threshold: influence of age, height, sex, and smoking and calculation of accurate centile values. Diabet Med 1991;8:157-61.

14 Drachman DA, Hart C. An approach to the dizzy patient. Neurology 1972;22:323-34.

15 Donofrio PD, Albers JW. AAEM Minimonograph 34 polyneuropathy: classification by nerve conduction polyneuropathy: classification by nerve conduction 889-903.

16 Hufschmidi A, Dichgans J, Mauritz KH, Hufschmidt $M$ Some methods and parameters of body sway quantification and their neurological applications. Archiv fü Psychiatrie und Nervenkaukheiten 1980;228:135-50.

17 Ojola M, Matikainen E, Groop E. Body sway in diabetic neuropathy. $¥$ Neurol $1985 ; 232$ (suppl): 188

18 Vrethem M, Ledin T, Ernerudh J, et al. Correlation between dynamic posturography, clinical investigation and neurography in patients with polyneuropathy. $O R L$ f Otorhinolaryngol Relat Spec 1991;53:294-8.

19 Ledin T, Odkvist L, Vrethem M, Moller C. Dynamic posturography in assessment of polyneuropathic disease. fournal of Vestibular Research 1991;1:123-8.

20 Black FO, Wall C, Nashner LM. Effects of visual and sup port surface orientation references upon postural contro port surface orientation references upon postural control in vestibular deficient

21 Solders G, Andersson T, Borin Y, Brandt L, Persson A. Electroneurography index: a standardised neurophysiological method to assess peripheral nerve function in patients with polyneuropathy. Muscle Nerve 1993;16: 941-6.

22 Harding AE, Thomas PK. The clinical features of hereditary motor and sensory neuropathy types I and II. Brain 1980;103:259-80.

23 McDonald WI. Physiological consequences of demyelination. In: Sumner AJ, ed. The physiology of peripheral nerve disease. Philadelphia: WB Saunders, 1980:265-86.

\section{Melkersson's syndrome}

When multiple sclerosis, sarcoidosis, leprosy, recurrent cranial polyneuritis, and other causes of recurring and sometimes bilateral lower motor neuron facial pareses have been considered there is a curious but rare group of cases which fit the picture of Melkersson's syndrome. In addition to facial palsy these patients have recurrent facial oedema, and about $25 \%$ have a congenitally horizontal fissuring of the tongue-lingua plicata. The first possible reference to this syndrome was that of Rossolimo in 1901 who described a woman with recurring facial palsy associated with attacks of migraine; but, this case may have been one of facial migraine.

Melkersson's paper, ${ }^{1}$ translated from Swedish, ${ }^{2}$ described a man of 35 who at the age of three had a left facial palsy which lasted for two weeks. At 14, facial palsy recurred on the same side, and again he recovered completely in three weeks. At 18 a third attack lasted about two weeks, but left residual weakness; he could not close the left eye tightly, and the left corner of his mouth did not move properly. At the age of 35 another attack was followed by severe and persisting paresis. After the age of 14, transient swelling occurred on the upper or lower lip. At first the swelling appeared with the facial palsy. When both facial palsy and swelling were present he felt unwell.
From the age of 35 his facial countenance was grotesque; the left half of his face was totally paralysed ... The left cheek was atrophic. Lower lip was cyanotic and intensely swollen and doughy, especially in the right half.

Melkersson thought a vasomotor dysfunction likely. He did not describe the lingua plicata, which is a condition affecting $0.5 \%$ of the population as an incompletely penetrant dominant trait. Rosenthal, a German neurologist, three years later referred to an abstract of Melkersson's paper in German, and noted lingua plicata in an arthritic family who also had Melkersson's syndrome of recurrent facial palsy and facial oedema. ${ }^{3} \mathrm{~A}$ familial occurrence has been confirmed in several subsequent reports.

$$
\begin{array}{r}
\text { J M S PEARCE } \\
304 \text { Beverley Road, } \\
\text { Anlaby, } \\
\text { Hull HU10 } 7 B G
\end{array}
$$

1 Melkersson E. Ett fall av recidiverande facialispares i samband med angioneurotiskt ödem. Hygiea 1928;90: band $\mathrm{737}-41$.

2 Furukawa T. Melkersson's syndrome. Neurology 1985;35: $1145-6$.

3 Rosenthal C. Klinisch-erbbiologischer Beitrag zur Konstitutionspathologie. Gemeinsames von (rezidivierender familiärer) Facialisähmung angioneurotischem Gesichtsödem und Lingua plicata in Arthritismus Familien. Z Gesamte Neurol Psychiatr 1931;131:475-501. 